\title{
Why Gravity a Weak Force of Nature
}

\author{
Kapil Chandra \\ University of Bastar, Dhararmpura 1, Jagdapur, India \\ Email: kapil.chandra@gov.in
}

How to cite this paper: Chandra, K. (2020) Why Gravity a Weak Force of Nature. Journal of High Energy Physics, Gravitation and Cosmology, 6, 353-356. https://doi.org/10.4236/jhepgc.2020.63028

Received: May 12, 2020

Accepted: July 5, 2020

Published: July 8, 2020

Copyright $\odot 2020$ by author(s) and Scientific Research Publishing Inc. This work is licensed under the Creative Commons Attribution International License (CC BY 4.0).

http://creativecommons.org/licenses/by/4.0/

\begin{abstract}
Mathematically we address, why gravity is a weak force of nature by proposing a force balance equation between classical force (gravity) and quantum force (strong interaction) while presuming that there is a natural balance between these forces. We reported, each side of the balance has two expressions of force where quantum force part consists of two types of quantum force with similar strength but classical force part has a constant force term and gravitational force where this constant force term has large numerical value thus higher weightage in the balance, however, gravity becomes weak itself to strike the balance.
\end{abstract}

\section{Keywords}

Gravity, Quantum Force, Gravitational Coupling Constant

\section{Introduction}

There are four types of interactions namely strong, weak, electromagnetic and gravity. The gravity is the oldest known force which is responsible for governing the universe at large scale, notwithstanding its weakest force of nature; particularly in number its weaker to strong interaction by 39 orders of magnitude nearly.

It has been a challenge to explain why the gravity is so weak and bothering physicists for years to answer. Another problem with this gravity is that this force is stand alone and not unified with other existing interactions of nature and becomes a hurdle in grand unification theory of natural interactions. Yet it is believed that quantum theory of gravity might address these open questions but this theory is in its evolving course, interested reader may refer to ref [1] and references therein for more detail.

\section{Relation between Classical and Quantum Force}

In order to explain the reason behind the weakness of gravity, here we come up 
with a different idea. We proposed an equation which shows the balance between known quantum and classical force which are dependent of mass and space. One can also say that it's a balance between short and long-range force. The proposed mathematical balance of force can be written as followings [2],

$$
\frac{h c}{R^{2}} \times \frac{m^{2} c^{3}}{h}=\frac{c^{4}}{G} \times G \frac{m^{2}}{R^{2}}
$$

equation is empirical and based on dimensional analysis anyway. Where all used constants hold its usual meanings and accepted numerical values. It is noticeable from the balance that space $R$ and mass $m$ is only used variable. In case of gravitational interaction we took $m^{2}$ i.e. similar mass instead of different mass as $m_{1}$, $m_{2}$ since in this paper we calculated the force for the same size of mass; i.e. for neutron \& proton which are relatively similar in mass.

In this force balance equation, all terms have the same dimension as force has thus denotes different forms of force and in this context, we call it Force Balance Equation (hereafter we abbreviated it as FBE).

\section{Quantum Form of Force}

The first two mathematical expression of this FBE has Planck's constant, therefore, it denotes quantum of force. Since, nuclear force is a form of quantum force [3] so that its numerical value can be estimated via these equations by replacing $R$ with size of nucleus and $m$ with the mass of proton or pion respectively.

Thus, the numerical value of strong force can be calculated from following expression,

$$
F=\frac{h c}{R^{2}}
$$

by substituting the numerical value of all used constant and variable $R$ as size of nucleus which is $R=10^{-15} \mathrm{~m}$ we obtained $F \sim 10^{+4} \mathrm{~N}$ or one can say its numerical value is nearly 4 orders of magnitude.

The second expression is as followings,

$$
F=\frac{m^{2} c^{3}}{h}
$$

this equation can calculate the amount of force which was mediated by pion as per Yukawa's meson theory since the mass is only used variable here. If we take $m$ as pion mass, this expression gives value which is same for what has been earlier calculated by Equation (2). This facilitates us very simple calculation of numerical amount of strong force. Theoretically estimated value in preceding paragraph is comparable to its predicted value by other established theory, however, our hypothesis is in consistent with other existing theory of physics.

Next, the other side of balance denotes classical force since both expression possess universal gravitational constant. The first expression denotes Planck force which is a constant force since it is independent of any variable, by substituting the numerical value if all constant it gives its large value in numbers [4]. 
The last term is well known classical gravity. For the proton-neutron at the nucleus the numerical value of gravitational force will be nearly $F \sim 10^{-35} \mathrm{~N}$. This makes clear that the numerical amount of gravity is weak with contrast to strong force that we calculated from mathematical expression of quantum force in preceding paragraph.

\section{Derivation of Gravitational Coupling Constant}

An important aspect of this $\mathrm{FBE}$ is that it gives the relative strength of strong force to gravity as written below,

$$
\frac{h c}{R^{2}}=\left(\frac{h c}{G m^{2}}\right) G \frac{m^{2}}{R^{2}}
$$

where one can denote,

$$
\alpha_{G}=\frac{h c}{G m^{2}}
$$

where $\alpha_{G}$ shows the relative strength of strong and gravitational force that is known as gravitational coupling constant. It suggests the gravity is weaker to strong force by 39 orders of magnitude [5]. Earlier this quantity was considered as a constant quantity and derived by comparing the gravity with energy but our hypothesis gives its systematic origin; this suggests our proposed FBE is in consistent with other existing theory.

\section{Why Gravity a Weak Force?}

Now, we come to the FBE again and found that unlike the numerical value of quantum force terms, the classical forces are not same; these are very asymmetric in numerical quantity. Since these both collectively incorporated in the natural balances of forces thus to retain the balance, gravity becomes weak itself. This might be the reason that gravity is weak force of nature. This is the mathematical interpretation of weakness of gravity.

The other possible reason behind the weakness of gravity might be that; the quantum force which is dependent of either mass or space is relatively strong but gravity is function of both variable, however, its a weak. Yet this fact is not clear that why gravity dependent of both mass and space; it may explain that fact. Apart from it, this hypothesis doesn't tell anything about why the gravity is long range force while its counterparts work at short range, this is a matter of future research.

\section{Conclusions}

In summary, we have shown, there is a numerical and dimensional balance between quantum and classical force where quantum side of balance consists of two different expressions of quantum force with same strength whereas the classical side is collectively incorporated from Planck force and classical gravity. This classical side of balance is dominated by the constant Planck force with 
large numerical value, however, gravity becomes weak itself to retain the balance mathematically. This might be the possible explanation why gravity is weak force of nature.

In other scenario, one can also justify the weakness of gravity by inferring that the constant force has large value and quantum expression of force which is either function of mass or space, has similar strength but lower to the constant Planck force whereas gravity is function of both variable so it becomes much weak. However, the idea is, gravity is dependent on both mass and space thus its comparably weak to other force. But, it is not clear how the variable have impact on the strength of force, its matter of future research.

\section{Acknowledgements}

I would like to say thanks to Prof. Dr. H.S. Tewari for fruitful discussions on this topic. We also thanks to the anonymous referee for his suggestions and comments to improve our understanding

\section{Conflicts of Interest}

The author declares no conflicts of interest regarding the publication of this paper.

\section{References}

[1] Rovelli, C. (2004) Quantum Gravity. Cambridge University Press, Cambridge. https://doi.org/10.1017/CBO9780511755804

[2] Chandra, K. (2019) Why Zeldovich Failed to Estimate the Precise Value of Cosmological Constant in Planck Unit? Journal of High Energy Physics, Gravitation and Cosmology, 5, 1098-1104. https://doi.org/10.4236/jhepgc.2019.54062

[3] Chandra, K. (2020) Does Hawking Predict the Correct Temperature of Black-Hole. Open Astronomy, 29, 1-3. https://doi.org/10.1515/astro-2020-0008

[4] Planck, M. (1899) Über irreversible Strahlungsvorgänge Sitzungsberichte der Königlich Preußischen Akademie der Wissenschaften zu Berlin (in German). 5, 440-480.

[5] Silk, J. (1977) Cosmogony and the Magnitude of the Dimensionless Gravitational Coupling Constant. Nature, 265, 710-711. https://doi.org/10.1038/265710a0 